\section{Similarity of nucleotide sequences at splicing sites}

SIR-We report an intriguing similarity between a sequence involved in processing of mitochondrial RNA and the consensus sequence at the 5 '-splice site of introns in transcripts of nuclear genes.

In Aspergillus nidulans, synthesis of mature mitochondrial RNA occurs by cleavage and end-trimming of large precursor molecules ${ }^{1-3}$. With one exception there are no significant similarities of sequence or structure between different processing sites. The exception concerns the sites where cleavages produce the $5^{\prime}$-termini of the mature $16 \mathrm{~S}$ and $23 \mathrm{~S}$ mitochondrial rRNA molecules. These sites lie in conserved 18-nucleotide motifs:

$$
\begin{aligned}
& 5^{\prime} \ldots \mathrm{GUU}_{\mathrm{GAA}}^{\mathrm{A}} \mathrm{UAUU}_{\mathrm{T}}^{\mathrm{A}} \mathrm{CA}- \\
& \text { AUAA } \overline{A G G U} \text { GAU } \ldots . . .3^{\prime}
\end{aligned}
$$

S1 nuclease analysis places the positions of the cleavage events in the quadruplet 5'-AGGU-3' indicated by the horizontal line.

The motif $5^{\prime}$-AGGU $\mathrm{G}^{\mathrm{A}} \mathrm{-3}^{\prime}$ also forms part of the consensus sequence for the $5^{\prime}$ splice junctions of introns in transcripts of nuclear genes ${ }^{4}$ :

$$
5^{\prime} \ldots \mathrm{A}_{\mathrm{AGGU}}^{\mathrm{C}} \mathrm{A} A \mathrm{AGU} \ldots 3^{\prime}
$$

The mitochondrial processing sequence therefore includes six adjacent nucleotides that conform with the splice junction consensus. Furthermore, cleavage of the mitochondrial sequence may occur at the same relative position as cleavage of the splice site.

It is unlikely that the similarity has arisen purely by chance. Conceivably, components of the A.nidulans nuclear splicing machinery (snRNA, for instance) also have unsuspected roles in processing mitochondrial RNA precursors. This attractive hypothesis is tempered by the fact that the $5^{\prime}$-splice junction consensus for A.nidulans nuclear transcripts differs from the general version shown above in that the exonic $5^{\prime}-\mathrm{C}_{\mathrm{AG}-3^{\prime}}$ sequence is absent ${ }^{5}$. Alternatively, the core sequence 5'-AGGU-3' may have an inherent disposition towards cleavage which aids RNA processing in both systems.

T.A. Brown Department of Biochemistry and Applied Molecular Biology,

UMIST,

Manchester M60 1QD, UK

N.J. DYSON

Amersham International,

Amersham HP7 9LL, UK

1. Dyson, N.J. thesis, Univ. Manchester (1988)

2. Brown, T.A. in Gene Structure in Eukaryotic Microbes (ed Kinghorn, J.R.) 141-162 (IRL, Oxford, 1987)

3. Dyson, N.J.et al. Gene (submitted).

4. Watson, J.D. et al. Molecular Biology of the Gene 4th edn (Benjamin/Cummings, Menlo Park, 1987)

5. Gurr. S.J., Unkles, S.E. \& Kinghorn, J.R. in Gene Structure in Eukaryotic Microbes (ed. Kinghorn. J.R.) 93-139 (IRL. Oxford, 1987).

\section{Incubation period of AIDS in haemophiliacs}

SIR-Ekert's assertion ${ }^{1}$, which has led to discussion $^{2.3}$, that haemophiliacs infected with human immunodeficiency virus (HIV) may carry a tenfold-lower risk of developing AIDS than HIV-infected individuals from other risk groups, is at odds with our own findings. As we reported at the recent European Conference of Clinical Aspects of HIV Infection, we are caring for a cohort of 395 HIV-infected haemophiliacs, of whom $38(10 \%)$ have developed AIDS so far. And in two large US cohorts ${ }^{4}, 12$ and $11 \%$ of HIV-infected haemophiliacs had developed AIDS by 1987. Thus it can be stated that the incidence of AIDS in HIV-infected haemophiliacs is not as low as proposed by Ekert.

With regard to the incubation period, information is difficult to obtain as the exact time of seroconversion is not known for most of the patients and as the number of HIV-infected patients in other risk groups is difficult to estimate ${ }^{3}$. During a mean follow-up period of 10 months, however, the disease progressed in 78 of 204 of our patients ( $38 \%$ ) according to the CDC-classification, so one must assume that the incidence of AIDS in this cohort will increase further. This is in accordance with Goedert et al ${ }^{5}$, who could not find a significant difference in the incidence of AIDS in cohorts of male homosexuals, intravenous drug-abusers and haemophiliacs.

T. KAMRADT

D. NIESE

B. KAMPS

Medizinische Universitätsklinik Bonn,

Klinische Immunologie,

S.-Freud-Strasse 25,

D-5300 Bonn 1, FRG

1. Ekert, H. Nature 329, 494 (1987)

. Turner, M.J., White, J.D. \& Souter, W.P. Nature 330, 702 (1987)

3. Rees, M. Nature 332, 312 (1988).

The Pennsylvania AIDS Surveillance Study Group Blood 70, 1208-1210 (1988)

5. Goedert, J.J. et al. Science 231, 992-995 (1986)

\section{Inheritance of insulin- dependent diabetes}

SIR-Todd et al.' have presented data to suggest that inheritance of insulindependent diabetes mellitus (IDDM) through the HLA region is recessive, that variation at the DQ locus is more important than that at the DR locus in IDDM pathogenesis, and further that variation at a single amino acid residue (position 57 of the $\mathrm{DQ}_{\beta}$ chain) protects an individual from IDDM. We show here that recessively inherited predisposition to IDDM, due to a dominant protective effect from aspartate occupying residue 57 , may be a misleading simplification.

Although the HLA-linked IDDM predisposition was initially described as recessive, investigators have since proposed more complex and realistic models ${ }^{2.3}$ which take into account the following observations: greatly increased risk of IDDM in DR3/4 heterozygotes; a tendency to dominant behaviour of DR4 in the absence of DR3, and recessive behaviour of DR3 in the absence of DR4; increased risk associated with DR1 in many studies; and the pronounced protective effect of DR2 (ref. 4). A picture is gradually emerging of a scale of susceptibility on which every allele, indeed almost every genotypic combination, occupies a distinct place. Furthermore, it is now understood that DR3 and DR4 play differing roles in IDDM pathology, suggesting that IDDM is a heterogeneous disease $^{5}$

It is difficult to reconcile the hypothesis of Todd et al. of susceptible and protective haplotypes based on a single $\mathrm{DQ}_{\beta}$ residue with the following observations. First, the DR7 haplotype, like the susceptibility haplotypes DR1, DR3 and DR4, lacks aspartate at position 57 of the $\mathrm{DQ}_{\beta}$ molecule. The theory of Todd et al. thus predicts DR7 to be a high-risk haplotype, whereas in fact it confers no additional risk to IDDM. They suggest this discrepancy might be explained by the unusual structure of the $\mathrm{DQ}_{a}$ chain encoded in the DR7 haplotype, and yet this structure is very similar to the $\mathrm{DQ}_{\alpha}$ chain encoded in the susceptible DR4 haplotype ${ }^{6}$. Second, in agreement with other studies, Todd $e t$ al. report that $\sim 10 \%$ of IDDM patients carry the protective aspartate residue in DQ, so that susceptibility to IDDM due to the absence of this residue could not be strictly recessive. Third, the statements of Todd et al. that the DR locus is not involved in IDDM are contradicted by recent experiments on NOD mice, which lack the DR equivalents of class II MHC molecules (the I-E genes). As Todd et al. point out, NOD mice, which spontaneously develop diabetes, lack aspartate at position 57 of the $\mathrm{I}-\mathrm{A}_{\beta}$ locus (equivalent to the human $\mathrm{DQ}_{\beta}$ ), unlike other mouse strains.

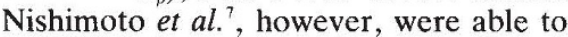
render the NOD mice non-diabetic by introducing the I-E genes, an experiment which suggests a role for the DR molecules in IDDM pathogenesis.

Current knowledge of the genetic contributions of the HLA region to IDDM strongly hints at several interactions: interaction within epitopes expressed on class II molecules, within single genes, within and between haplotypes, and finally interactions with unlinked loci and the non-genetic environ-

1. Todd, J.A. et al. Nature 329, 599-604 (1987)

2. Rotter. J.I. et al. Diabetes 32, 169-174 (1983)

3. Louis. E.J. \& Thomson. G. Diabetes 35. 958-963 (1986)

4. Thomson, G. Am. J. hum. Genet. 36, 1309-1317 (1984)

5. Ludvigsson. L. et al. Diabetologia 29. 207-210 (1986).

6. Moriuchi, J. et al. Proc. natn. Acad. Sci. U.S.A. 82, 34203424 (1985)

7. Nishimoto. H. et al. Nature 328. 432-434 (1987). 